\title{
The Role of CT-scan Studies for the Diagnosis and Therapy of Acute Respiratory Distress Syndrome
}

\author{
Luciano Gattinoni, MD, FRCP*, Pietro Caironi, MD, \\ Franco Valenza, MD, Eleonora Carlesso, MSc \\ Istituto di Anestesiologia e Rianimazione, Dipartimento di Anestesia, Rianimazione, e Terapia del Dolore, \\ Fondazione IRCCS-Ospedale Maggiore Policlinico, Mangiagalli, Regina Elena di Milano, \\ Università degli Studi di Milano, Via F. Sforza 35, 20122, Milano, Italy
}

The radiologic assessment of lung infiltrates by conventional anterior-posterior chest radiographs has been embedded in the concept of acute respiratory distress syndrome (ARDS) since its original description by Ashbaugh and colleagues [1]. In fact, the presence of bilateral lung infiltrates as detected by chest radiograph, in association with "refractory" hypoxemia and impaired mechanics of the respiratory system (stiff lung), was one of the mandatory criteria for ARDS diagnosis. In the mid-1980s, however, the first reports describing ARDS by using CT scanning changed the view of the syndrome [2-4]: the ARDS lung appeared to be affected by the disease process nonhomogeneously, with the CT densities distributed mainly in the dependent lung regions. The quantitative analysis of CT scanning gave new insights concerning the pathophysiology of the syndrome and provided a firm rationale for modifying the mechanical ventilation in use at that time [5-7]. This article discusses in some detail what the authors believe is or should be the role of the CT scanning in the diagnosis and therapy of ARDS.

\section{Quantitative analysis of CT scanning}

Because the authors believe that the quantitative analysis of CT scanning is of paramount

\footnotetext{
* Corresponding author. Istituto di Anestesiologia e Rianimazione, Fondazione IRCCS-"Ospedale Maggiore Policlinico, Mangiagalli, Regina Elena” di Milano, Via Francesco Sforza, 3520122 Milano, Italy.

E-mail address: gattinon@policlinico.mi.it (L. Gattinoni).
}

importance for ARDS diagnosis and for a rational setting of mechanical ventilation, a brief description of the technique and its limits is necessary. (A more detailed description of the quantitative analysis may be found elsewhere $[7,8]$ ).

\section{Physical principles of CT scanning}

The digital image produced by the CT scan is based on the measure of the attenuation coefficient $(\mu)$ (ie, the reduction of the radiation intensity upon passage through matter). Traversing from one side of the patient to the other, the X-ray beam is attenuated by all the voxels (volume elements of the tissue) through which passes. The intensity of the emerging X-ray is described by the Lambert law of absorption, that is,

$$
I=I_{0} e^{\int \mu d l}
$$

where $I$ is the intensity of the emerging X-ray beam, and $I_{0}$ is the intensity of the X-ray beam at the source. By measuring the intensity $I$ and $I_{0}$, the CT calculates the integral over the function $\mu$ of the attenuation coefficient along the X-ray beam. Then, through a different mathematical algorithm (usually filtered back projection), a given attenuation number $\mu$ is assigned to each voxel $\left(\mu_{\text {voxel }}\right)$. This attenuation number primarily represents the density of the voxel (ie, the ratio of mass to the volume) and is expressed as the CT number [9], which relates to the density of the water $\left(\mu_{\text {water }}\right)$ :

$$
C T=1000 \times \frac{\mu_{\text {voxel }}-\mu_{\text {water }}}{\mu_{\text {water }}}
$$

Conventionally a CT number equal to zero Hounsfield units (HU) indicates that density equals 
that of water; a CT number equal to $-1000 \mathrm{HU}$ indicates a density similar to that of gas, and a CT number equal to $+1000 \mathrm{HU}$ indicates a density equal to that of bone. Of note, the CT number assigned to a voxel does not identify the material of the voxel but simply expresses its density as related to the density of water.

\section{Computation of gas and tissue volume}

Assuming the specific weight of the lung tissue as equal to 1 (ie, equal to the specific weight of water), it is possible to compute from the density of the voxel the volume of gas and the volume of tissue, both in the whole lung and in a particular region of interest $[5,6]$. In fact, a voxel with a CT number equal to $0 \mathrm{HU}$ will be composed only of tissue, a voxel with CT number equal to -1000 HU will be composed only of gas, and a voxel with a CT number equal to $-700 \mathrm{HU}$ will be composed of $30 \%$ tissue and $70 \%$ gas. Therefore, by knowing the CT number and the volume of voxels of the whole lung, or of a given region of interest, it is possible to compute the gas and the tissue volumes, according to the following formulas:

$$
\begin{aligned}
\text { Volume } / \text { weight of tissue }= & \left(1-\frac{C T}{-1000}\right) \\
& \times \text { Total volume }
\end{aligned}
$$

\section{Volume of gas $=$ Total volume}

\section{- Volume of tissue}

From these basic formulas it is possible to derive several other variables that are important in understanding the pathophysiology of ARDS. In particular, the excess tissue mass [10] may be estimated by referring to the ideal tissue weight and the ideal average CT number of the whole lung in normal subjects:

\section{Excess Tissue Mass $=$ Actual Tissue Mass

$$
\text { - Ideal Tissue Mass }
$$

To estimate the hypothetical normal tissue of a given patient, it is possible to estimate the expected functional residual capacity in the supine position, with sedation, and to assign an average normal CT number. Functional residual capacity can be found using the formula proposed by Ibanez and colleagues [11], which refers to supine patients; for an average CT number the authors use $-654 \mathrm{HU}$ (derived from normal subjects) [12]. The estimation of the ideal tissue mass may be improved when a larger database of normal subjects becomes available and has been examined.

Another variable that may be important when setting mechanical ventilation is the superimposed pressure $[12,13]$. This parameter represents the hydrostatic pressure that develops into the lung parenchyma from the nondependent to the dependent regions. Its computation assumes that the pressure is transmitted through the lung parenchyma as in a fluid. Accordingly, knowing the density and the height of the lung, it is possible to compute for at any point along the vertical axis (the sternovertebral axis in the supine position) the pressure exerted by the lung parenchyma lying above.

$$
\begin{aligned}
& \text { Superimposed Pressure }=\left(1-\frac{C T}{-1000}\right) \\
& \times \text { height }_{\text {level }}+\text { Superimposed }_{\text {Pressure }} \text { above level }
\end{aligned}
$$

\section{Lung compartments}

To investigate better the lung functional anatomy and its variations in ARDS as detected by CT scan, it is convenient to refer to the lung compartments, defined according to their specific degree of aeration in normal lungs. When examining the frequency distribution of the CT numbers in normal lungs, it is easy to recognize a hyperinflated compartment (which includes voxels with a CT number between $-1000 \mathrm{HU}$ and -901 HU), a normally aerated compartment (which includes voxels with a CT number between -900 $\mathrm{HU}$ and $-501 \mathrm{HU})$, a poorly aerated compartment (which includes voxels with CT number between -500 and $-101 \mathrm{HU}$ ), and a nonaerated (or gasless) compartment (which includes voxels with a CT number between $-100 \mathrm{HU}$ and $+100 \mathrm{HU}$.) These arbitrary thresholds, which were introduced in 1987 by the authors' group [14], are the most widely accepted in the literature, although other authors have proposed slightly different thresholds [7].

The division of lung parenchyma into compartments with different degrees of aeration is particularly useful when assessing the difference in functional lung anatomy caused by application of different tidal volumes or airway pressures. In fact, the division of lung parenchyma into different compartments makes it possible to estimate the efficacy of higher airway pressures in opening or reversing lung atelectasis (ie, to estimate lung recruitment). The lung recruitment occurring between two different levels of airway pressures may be estimated by computing the amount of gasless tissue regaining aeration going from the 
lower to the higher level of pressure [15]. The author prefer to express the lung recruitment after standardization for the total weight of the lung:

Lung Recruitment

$$
=\frac{\text { Gasless }_{\text {tissue }} \text { paw } 1-\text { Gasless tissue }_{\text {paw2 }}}{\text { Total Lung Weight }}
$$

where Paw1 and Paw2 are, respectively, the lower and the higher applied airway pressure [16].

\section{CT scanning and diagnosis of acute respiratory distress syndrome}

Since its original description by Ashbaugh and colleagues [1], several clinical definitions of ARDS have been proposed. The key endeavor, always, has been to find clinical and easily measurable signs that could identify the primarily pathologic characteristics of ARDS (ie, the high-permeability inflammatory noncardiogenic lung edema). The symptoms identified originally by Ashbaugh and colleagues were hypoxemia refractory to increased inspired fraction of oxygen $\left(\mathrm{FIO}_{2}\right)$, bilateral pulmonary infiltrates as detected by chest radiograph, low compliance of the respiratory system, and, at autopsy, an increased lung weight [1]. Subsequently, Murray and colleagues [17] proposed a lung injury score for ARDS definition. This score included a progressively decreasing ratio of partial pressure of arterial oxygen $\left(\mathrm{PaO}_{2}\right)$ to $\mathrm{FIO}_{2}$ $\left(\mathrm{PaO}_{2} / \mathrm{FIO}_{2}\right)$, different degrees of consolidation on chest radiograph, and a progressively decreased compliance of the respiratory system. The most widely accepted and employed definition of ARDS, however, is the one proposed by the American-European Consensus Conference, which relies primarily on hypoxemia $\left(\mathrm{PaO}_{2} / \mathrm{FIO}_{2}<300\right)$ and the presence of bilateral infiltrates [18]. A more recent definition has been proposed through a Delphi method $[19,20]$. Once again this definition relies primarily on hypoxemia $\left(\mathrm{PaO}_{2} / \mathrm{FIO}_{2}<200\right.$ with a positive end-expiratory pressure $[\mathrm{PEEP}] \geq$ $10 \mathrm{~cm} \mathrm{H}_{2} \mathrm{O}$ ) and on the presence of bilateral pulmonary infiltrates as detected by chest radiograph. All these definitions exclude the presence of a unilateral lung injury, as well as the presence of cardiogenic edema as assessed by clinical judgment or by measurements of the pulmonary occlusion pressure. The diagnostic accuracy of these different definitions has been assessed recently, using as a reference standard the finding of diffuse alveolar damage on autopsy [21]. The results have been rather disappointing, however. The three different definitions, in fact, resulted in a quite poor sensitivity and specificity (see Table 1). In particular, the most commonly used criteria for ARDS diagnosis, as assessed by the American-European Consensus Conference, showed an acceptable sensitivity $(0.83 \%)$ but a quite poor specificity $(0.51 \%)$. The correct identification of ARDS is still an open issue, and the clinical consequences may be important. In fact, every attempt to improve survival during this syndrome by testing different ventilatory or therapeutic strategies (such as different tidal volumes, PEEP levels, different drugs, or other means) is based on randomized clinical trials that enrolled patients according to a given

Table 1

Different definitions of acute respiratory distress syndrome*

\begin{tabular}{|c|c|c|c|c|c|}
\hline Study & Hypoxemia $^{a}$ & $\begin{array}{l}\text { Infiltrates on } \\
\text { chest radiographs }\end{array}$ & $\begin{array}{l}\text { Positive } \\
\text { end-expiratory } \\
\text { pressure }\end{array}$ & $\begin{array}{l}\text { Compliance of } \\
\text { respiratory } \\
\text { system }\end{array}$ & $\begin{array}{l}\text { Lung } \\
\text { weight }\end{array}$ \\
\hline Ashbaugh et al [1] & Yes & Yes & No & Yes & Yes \\
\hline Murray et al [17] & Yes (graded 1-4) & Yes (graded 1-4) & Yes (graded 1-4) & Optional & No \\
\hline $\begin{array}{l}\text { American-European } \\
\text { Consensus } \\
\text { Conference [18] }\end{array}$ & Yes & Yes & No & No & No \\
\hline Ferguson et al [20] & Yes & Yes & Yes & No & No \\
\hline
\end{tabular}

* Most of the definitions proposed do not consider respiratory mechanics or carbon dioxide exchange (such as dead space). Moreover, none of these definitions takes into account the lung permeability or lung edema, the primary characteristics of the lung in acute respiratory distress syndrome.

${ }^{a}$ Hypoxemia was determined according to the ratio of partial pressure of arterial oxygen to fraction of inspired oxygen $\left(\mathrm{PaO}_{2} / \mathrm{FIO}_{2}\right)$.

(Data from Ferguson ND, Frutos-Vivar F, Esteban A, et al. Acute respiratory distress syndrome: underrecognition by clinicians and diagnostic accuracy of three clinical definitions. Crit Care Med 2005;33(10):2228-34.) 
definition. It is evident that an inappropriate definition of ARDS and a consequent inappropriate enrolment may lead to biased results and lead to the rejection of techniques that might be potentially useful in ARDS patients.

How does the CT imaging fit in this general framework? All the present definitions of ARDS include the presence of bilateral pulmonary infiltrates. Actually, these findings should represent a surrogate of the direct measurement of the inflammatory lung edema. Unfortunately, the infiltrates seen on chest radiograph may derive from various conditions, such atelectasis, interstitial edema, intra-acinar edema, or consolidation [7]. In clinical practice radiographs are often obtained in varying technical conditions (eg, voltage, distance, degree of inflation). Even the concept of bilateral infiltration is questionable, as shown by Meade and colleagues [22]. Indeed, the specificity of chest radiograph in indicating the generalized inflammatory edema that is the hallmark feature of ARDS is highly questionable. CT scanning and, particularly its quantitative analysis, may be a much better indicator of lung edema.

The authors have recently analyzed CT scans of 68 patients affected by acute lung injury (ALI) or ARDS, as defined by the American-European Consensus Conference. For comparison, they analyzed CT scans from 34 patients who had unilateral pneumonia and 39 patients who had healthy lungs [16]. Regarding the definition of ARDS, it may be interesting in these groups of patients to examine the total lung weight, which reflects the amount of inflammatory lung edema. As shown in Fig. 1, patients who had unilateral pneumonia had a significantly higher lung weight than patients who had normal lungs and significantly lower lung weights than patients who had ARDS. After the authors identified patients who had ALI/ARDS according to their lower or higher lung recruitability, however, they observed that patients who had lower lung recruitability (below $9 \%$

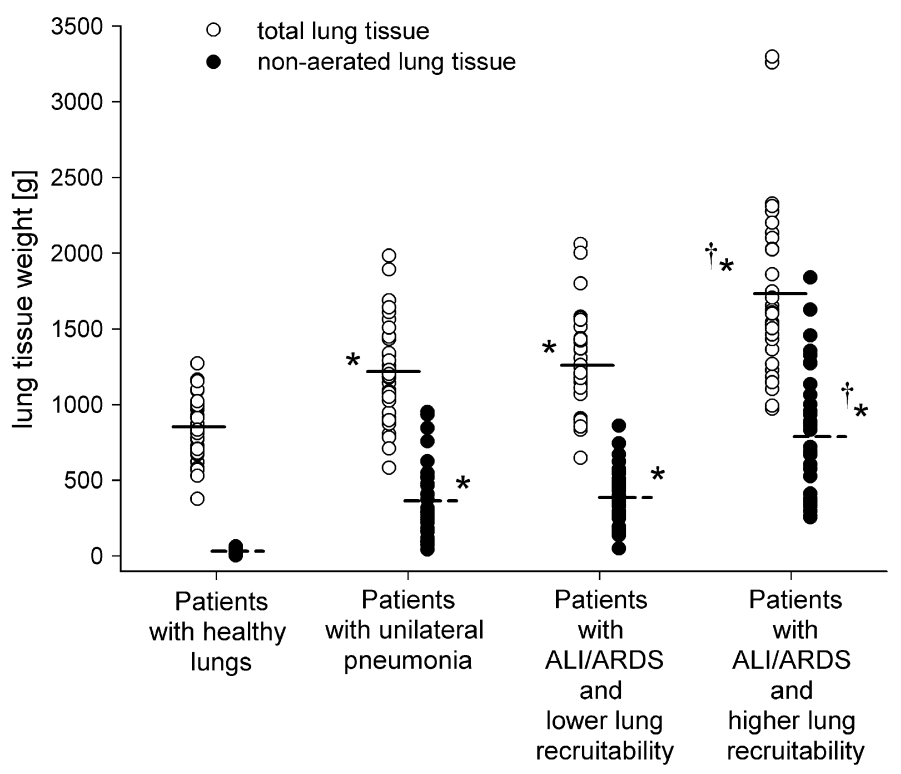

Fig. 1. The weights of total lung tissue and nonaerated lung tissue in 39 patients who had healthy lungs, 34 patients who had unilateral pneumonia, 34 patients who had ALI/ARDS with a lower amount of lung recruitability (at or below the overall median value of $9 \%$ ), and 34 patients who had ALI/ARDS with a higher amount of lung recruitability. Data for the patients who had healthy lungs and unilateral pneumonia were obtained from whole-lung CTs obtained for diagnostic purposes. Data for patients who had ALI or ARDS were obtained from a CT scan of the whole lung performed at a PEEP of $5 \mathrm{~cm} \mathrm{H}_{2} \mathrm{O}$. Solid lines represent mean values of total lung weight; dashed lines represent mean values of nonaerated lung tissue weight. ALI, acute lung injury; ARDS, acute respiratory distress syndrome; *, $P<.01$ for the comparison with patients who had healthy lungs; $\dagger, P<.01$ for the comparison between the groups of patients who had ALI or ARDS and a higher percentage of potentially recruitable lung and the other three groups. (From Gattinoni L, Caironi P, Cressoni $\mathrm{M}$, et al. Lung recruitment in patients with the acute respiratory distress syndrome. N Engl J Med 2006;354(17):1784; with permission. Copyright () 2004 Massachusetts Medical Society, all rights reserved.) 
of the total lung weight, the median value of the entire ALI/ARDS population) had the same lung weight as patients who had unilateral pneumonia (who, by definition, are not considered to have ARDS). In contrast, patients who had higher lung recruitability (greater than $9 \%$ of the total lung weight) showed a significantly higher lung weight. These findings emphasize how the issue of ALI/ARDS definition may be critical. If the hallmark characteristic of ARDS is lung edema (and, therefore, the total weight of the lung), either patients who have unilateral pneumonia should be included in ALI/ARDS definition, or patients who have lower lung recruitability should be excluded.

CT scans taken both at end-expiration, with low levels of PEEP, and at end-inspiration, at an airway pressure of $45 \mathrm{~cm} \mathrm{H}_{2} \mathrm{O}$, allow a precise definition of lung recruitability. Moreover, they can be used to compute the fraction of the lung that is consolidated (ie, the fraction of lung tissue that does not regain aeration even at $45 \mathrm{~cm} \mathrm{H}_{2} \mathrm{O}$ airway pressure). Table 2 shows the lung composition of patients who had ALI/ARDS grouped according to the amount of lung recruitability. As shown, the core disease, represented by the "consolidated" lung tissue, is nearly identical in patients who have higher or lower lung recruitability, accounting for about $24 \%$ of the total lung weight. The difference lies in the amount of the recruitable lung, which represents, in essence, the portion of lung tissue that is collapsed and capable of being opened. Lung collapse, then, is likely to be a function of the gravity-dependent compressive forces (the superimposed pressure) generated by the increased lung mass (ie, lung edema) $[13,15,23]$. Indeed, a possible model of ARDS pathophysiology may include an equally represented portion of core disease (the "consolidated" lung tissue), for instance a regional pneumonia. In some patients, the spreading of the inflammatory reaction is limited, leading to a lower amount of lung edema and lung collapse and, therefore, to a lower amount of lung recruitability. In contrast, in other patients the spreading of the inflammatory reaction is much greater, resulting in a more diffuse edema, an increased superimposed pressure with gravity-dependent lung collapse, and, therefore, greater lung recruitability (Fig. 2). At the moment, it is not known why the original lung disease remains compartmentalized in some patients and in others the compartmentalization of the disease is lost. This loss of compartmentalization may be caused by a different local balance between proinflammatory and anti-inflammatory molecular pathways or by inappropriate and injurious mechanical ventilation. Whatever the mechanisms, the authors strongly believe that the definition of ARDS should be limited to patients who have higher lung recruitability (ie, to patients who have generalized noncompartmentalized inflammatory lung edema). Mortality at discharge from ICUs was observed to be significantly associated with the extent of the inflammatory reaction and not with the core disease. This observation is surprisingly similar to what is known about sepsis: what is strictly linked with mortality is not the original and local infection but the spreading of the inflammatory

Table 2

Functional anatomy as detected by CT scan in patients who have acute lung injury/acute respiratory distress syndrome according to the amount of lung recruitability

\begin{tabular}{|c|c|c|c|}
\hline Lung characteristics & Lower lung recruitability $^{\mathrm{a}}$ & Higher lung recruitability & P-value \\
\hline Lung weight (g) & $1266 \pm 327$ & $1735 \pm 547$ & $<.001$ \\
\hline Amount of open lung $(\%)^{\mathrm{a}}$ & $70 \pm 12$ & $56 \pm 16$ & $<.001$ \\
\hline $\begin{array}{l}\text { Amount of lung } \\
\text { recruitability }(\%)^{\mathrm{b}}\end{array}$ & $5 \pm 4$ & $21 \pm 10$ & $<.001$ \\
\hline $\begin{array}{l}\text { Amount of consolidated } \\
\text { lung }(\%)^{\mathrm{c}}\end{array}$ & $25 \pm 12$ & $24 \pm 14$ & .69 \\
\hline
\end{tabular}

\footnotetext{
${ }^{\mathrm{a}}$ The amount of open lung denotes the portion of lung parenchyma with a density between $-101 \mathrm{HU}$ and $-1000 \mathrm{HU}$, as detected by CT scan.

${ }^{b}$ Lung recruitability was defined as the proportion of lung tissue in which aeration was restored at airway pressures from $5-45 \mathrm{~cm} \mathrm{H}_{2} \mathrm{O}$.

${ }^{c}$ Consolidated lung tissue was defined as the proportion of lung tissue that was not openable even at an airway pressure of $45 \mathrm{~cm} \mathrm{H}_{2} \mathrm{O}$.

(Data from Gattinoni L, Caironi P, Cressoni M, et al. Lung recruitment in patients with the acute respiratory distress syndrome. N Engl J Med 2006;354(17):1775-86.)
} 


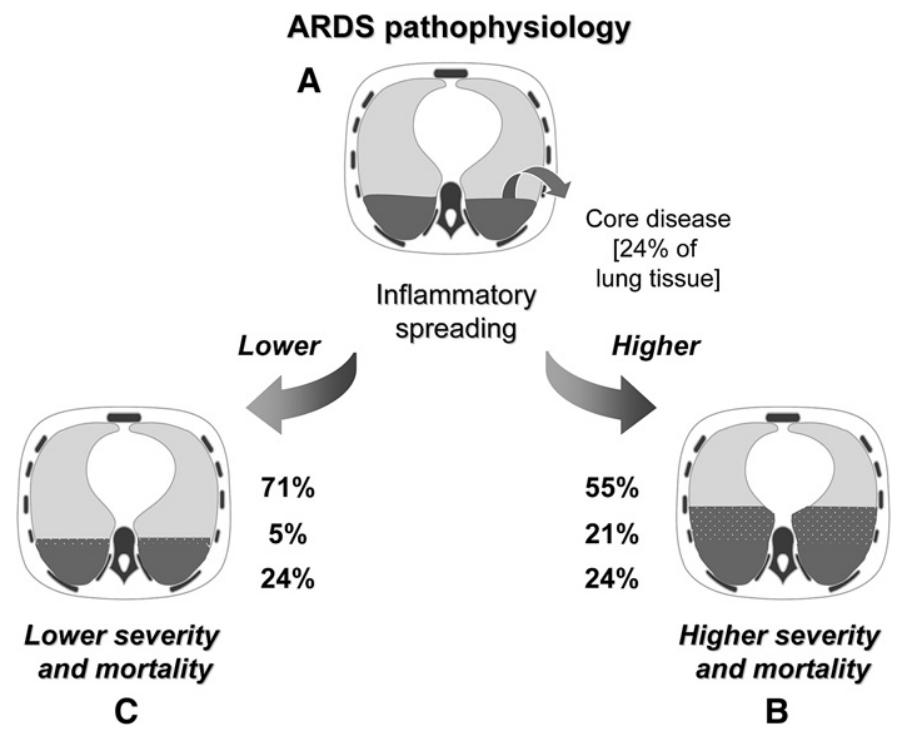

Fig. 2. Theoretical model of the pathophysiology of ARDS and its association with lung recruitability. As shown in $(A)$, about $24 \%$ of total lung weight that seems incapable of being opened ("consolidated") may represent the core disease (eg, for instance, a pneumonia focus). (B) Because of different biologic mechanisms or injurious ventilatory settings of mechanical ventilation, some patients lose the compartmentalization of the initial injury, showing a widespread inflammatory reaction involving the remaining part of the lung parenchyma. This inflammation will lead to greater lung collapse, higher lung recruitability, and higher mortality. (C) In contrast, other patients maintain the compartmentalization of the original disease. As a consequence, the lung edema and alveolar collapse are lower, determining lower lung recruitability, and leading to a higher survival.

reaction. If the ARDS is defined as the condition detected in patients who have higher lung recruitability, the extraordinary potential of CT analysis in defining ARDS is immediately apparent. The notion that lung edema or lung capillary permeability should be a cornerstone of the definition of ARDS is not novel [24,25], but, unfortunately, it has been ignored for long time, probably because of the technical difficulties associated with its assessment. The authors believe that two CT scans are required for ARDS diagnosis, one taken at end-expiration at low pressure level, and one taken at end-inspiration at a higher pressure level. It is conceivable that CT scanning of the whole lung is not necessary for this purpose, and that the combination of only three lung CT slices (taken at the lung apex, hilum, and basis) may be sufficient for the assessment of lung recruitability and therefore for the identification of ARDS.

\section{CT scanning and therapy for acute respiratory distress syndrome}

The therapy for ARDS is multifactorial, because it includes the specific therapy for the injury leading to ARDS, possible interventions in some of the molecular pathways leading to the widespread inflammation or coagulation abnormalities (eg, steroids, activated protein C), and, finally, supportive therapy, which primarily includes respiratory support with mechanical ventilation. The authors believe that CT scanning is an essential tool for the assessment of some of the effects that mechanical ventilation may cause on the lung parenchyma (resulting in the ventilatorinduced lung injury), and, more important, is the most objective technique now available for a rational setting of mechanical ventilation.

\section{Diagnosis of complications of mechanical ventilation}

It is well recognized and widely documented that the use of CT scanning during ARDS may provide information that cannot be derived from the conventional anterior-posterior chest radiographs. Consequently, it has been shown that the information gained from CT scanning frequently affects patient clinical management [26-28]. For example, in a retrospective review of 74 patients who had ARDS, CT scanning yielded additional 
information in $66 \%$ of the population and had a direct influence on treatment of $22 \%$ [26]. Entities that can be detected more often in CT scans than in chest radiographs are pleural effusions, lobar atelectasis, lung abscesses, lung bullae, and occult pneumothoraces, typically in the anterior, nondependent lung regions.

The authors believe that one of the primary indications for CT scanning of patients who have ARDS is a sudden and unexplained deterioration of the clinical status or the lack of expected improvement. In these circumstances, an occult complication, when present, may be detected by CT scanning.

\section{Assessment of lung injury severity and lung recruitability}

Although in ARDS CT scanning is not yet a routine clinical practice, the authors believe it may be the best tool to assess the severity of lung injury and lung recruitability. It is important to remember how the pulmonary units (the acini) and the CT pulmonary units (the voxels) are matched $[7,8]$. The volume of a normal pulmonary acinus, at end-expiration, ranges from 16 to $22 \mathrm{~mm}^{3}$, including about 200 alveoli [29]. Because about $10 \%$ of this volume is represented by tissue, it follows that, if the acinus is gasless, its volume will be about $2 \mathrm{~mm}^{3}$. On the other hand, the "CT pulmonary unit" (ie, the voxel) may have different dimensions. In most ARDS studies, in which the axial thickness was $10 \mathrm{~mm}$ and the matrix size $256 \times 256$, the volume of the voxel has equaled $22.5 \mathrm{~mm}^{3}(1.5 \mathrm{~mm} \times 1.5 \mathrm{~mm} \times 10$ $\mathrm{mm}$ ), which matches the dimensions of a normal acinus at end-expiration. If the acini are gasless, however, up to 10 acini are included in a voxel, whereas at total lung capacity only half an acinus is included. Actually, if the acinus is flooded, its volume will equal the voxel volume. The most recent scanners, such as the 16-slice CT scanners, however, use an approximately cubic voxel, with a dimension equal to 0.22 to $0.34 \mathrm{~mm}^{3}$, including just $1 \%$ of normal acinus (ie, about 20 alveoli).

These technical notes underline the importance of the quantitative analysis. In fact, a pure lung atelectasis (each voxel including about 20 acini) cannot be differentiated, per se, from pure lung edema (1 voxel including about 1 acinus or less). The quantitative analysis of the entire lung slice or of the whole lung parenchyma may easily differentiate the two conditions, however, because a normal weight will indicate lung atelectasis, whereas an increased weight will indicate lung edema. Moreover, phenomena such as lung recruitment can be quantified by obtaining two CT scans in two different standard conditions, as explained previously.

\section{Assessment of lung protection and setting the tidal volume}

Taking a CT scan at end-expiration, in standard conditions at a low level of PEEP, makes it possible to quantify the lung weight and the amount of nonaerated tissue as well as the amount of normally aerated, poorly aerated, and hyperinflated lung tissue. As shown in Fig. 3, patients included in the current definition of ALI/ARDS showed a fraction of nonaerated tissue (collapsed plus consolidated lung tissue) that ranged from $5 \%$ to $10 \%$ of the total lung weight to as much as $70 \%$ to $80 \%$ of total lung weight. These findings suggest that the lung tissue open to ventilation (poorly aerated plus normally aerated lung tissue) may range from $95 \%$ to $30 \%$ of the total lung weight. Therefore two different considerations may be observed. First, the overall lung injury, per se, varies widely throughout ALI/ ARDS population. Of note, mortality at discharge from ICU was $12 \%$ in patients who had a fraction of gasless tissue lower than the median value (37\% of the total lung weight) but was $44 \%$ $(P<.01)$ in patients who had a fraction of gasless tissue higher than the median value. Second, the portion of the lung exposed to the potentially harmful effect of ventilation (ie, the fraction of open lung) may vary from $95 \%$ to $30 \%$ of the lung weight.

Within this framework it is possible to discuss the rationale for using different-sized tidal volumes. There is convincing evidence that the use of high tidal volumes $(12 \mathrm{~mL} / \mathrm{kg}$ of predicted body weight) results in increased mortality as compared with the use of low tidal volumes $(6 \mathrm{~mL} / \mathrm{kg}$ of predicted body weight) [30]. Unfortunately, the tidal volumes tested in several randomized trials were normalized by the body weight (actual or ideal) [30-34]. This standardization was introduced, although not explicitly stated, as a surrogate for standardization of the lung size. CT scans, however, showed that, for the same body weight, the portion of lung open to ventilation may be extremely variable, and no relationship was found between the predicted body weight and the portion of the lung open to ventilation. For example, for a male patient who has ARDS and who has a predicted body weight of about $70 \mathrm{~kg}$, the recommended tidal 


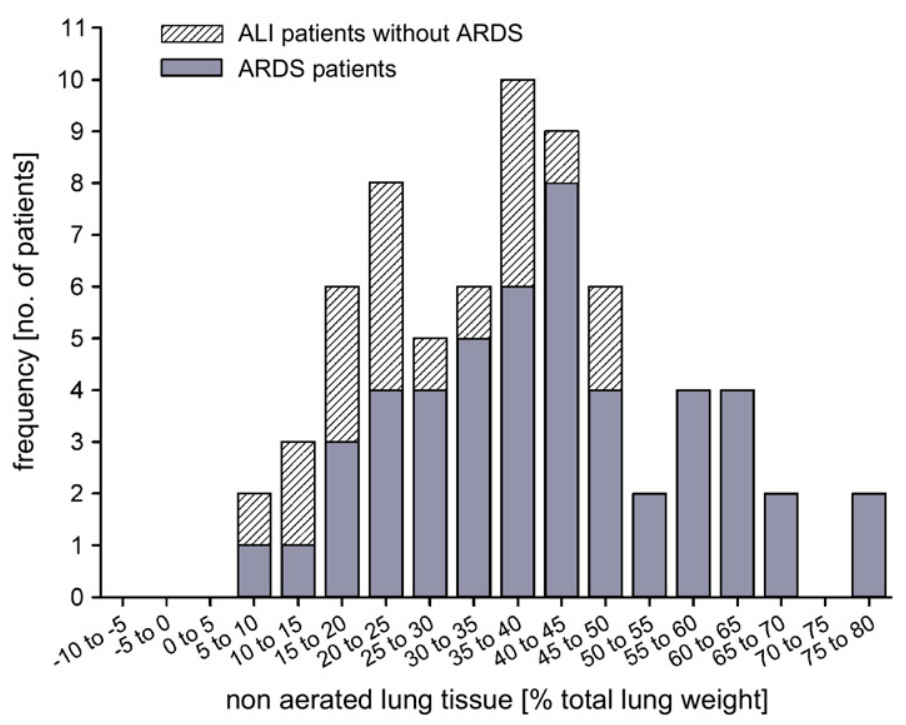

Fig. 3. The frequency distribution of the nonaerated lung tissue measured by CT scan at $5 \mathrm{~cm}$ of $\mathrm{H}_{2} \mathrm{O}$ PEEP in the overall study population $(n=68)$, expressed as a proportion of the total lung weight. Dashed columns represent patients classified as affected by ALI without ARDS $\left(\mathrm{PaO}_{2} / \mathrm{FIO}_{2}<300\right)$. Gray columns represent patients classified as affected by ARDS $\left(\mathrm{PaO}_{2} / \mathrm{FIO}_{2}<200\right)$. The nonaerated lung tissue was defined as the lung tissue having a physical density at CT scan image analysis between $+100 \mathrm{HU}$ and $-100 \mathrm{HU}$, representing the portion of lung parenchyma that is consolidated plus the portion of lung parenchyma that is collapsed (ie, the lung injury severity). ALI, acute lung injury; ARDS, acute respiratory distress syndrome; $\mathrm{PaO}_{2} / \mathrm{FIO}_{2}$, ratio of partial pressure of arterial oxygen to fraction of inspired oxygen; PEEP, positive end-expiratory pressure. (Reprinted from the online Supplementary Appendix of Gattinoni L, Caironi P, Cressoni $\mathrm{M}$, et al. Lung recruitment in patients with the acute respiratory distress syndrome. N Engl J Med 2006;354(17):1775-86; with permission. Copyright (C) 2004 Massachusetts Medical Society, all rights reserved.)

volume would be $420 \mathrm{~mL}$. This volume might be applied to a lung parenchyma actually open up to $70 \%$ (ie, $1750 \mathrm{~mL}$ ), creating an alveolar strain (the ratio between the applied tidal volume and the lung volume to which the tidal volume is applied) of about 0.24 [35]. If, however, the open lung is $50 \%$, or only $30 \%$, alveolar strain would increase to 0.34 or 0.56 , respectively. Because the main trigger of ventilator-induced lung injury is nonphysiologic excessive alveolar strain, it is clear that tidal volume should be tailored to the actual open portion of the lung parenchyma to prevent the further development of the inflammatory reaction and lung injury. It follows that when the amount of open lung parenchyma is great enough, a tidal volume greater than $6 \mathrm{~mL} / \mathrm{kg}$ predicted body weight may be safe, and by applying such a tidal volume it may be possible avoid the use of heavy sedation and paralysis often necessary during a very low tidal volume ventilation. In contrast, when the open fraction of lung parenchyma is very small, even a tidal volume of $6 \mathrm{~mL} / \mathrm{kg}$ predicted body weight may induce an excessive nonphysiologic alveolar strain. In this case, alternative modes to support the respiratory function (eg, high-frequency oscillatory ventilation, extracorporeal lung assist, and other means) should be sought.

\section{Assessment of lung recruitability and setting positive end-expiratory pressure}

Although the reduction of tidal volume, allowing a more gentle ventilatory treatment of the lung, probably reduces the global nonphysiologic strain on the lung regions open to ventilation, in theory the application of higher levels of PEEP maintain open lung regions that have been opened during inspiration (recruitment) but that would otherwise collapse at end-expiration [15,36]. Indeed, the possible beneficial effects of higher PEEP levels rely on the prevention of the intratidal collapse and decollapse of a portion of the lung [37]. Because this phenomenon produces local excessive stress and strain on the structures at the interface between the closed and the open portion of the lung, high levels of PEEP putatively will be effective by preventing the regional excessive stress and strain. At the same time, because 
the PEEP is an airway pressure applied to the lung parenchyma, it will increase the stress and strain of the already open lung regions, usually located in the nondependent portion of the lung. Although extensive experimental data have shown that PEEP may prevent ventilator-induced lung injury [38-40], the experimental models of ARDS (ie, involving oleic acid or lung lavage) usually are characterized by great lung recruitability, but in human patients lung recruitment is not always possible. Moreover, despite its physiologic rationale, the application of higher PEEP did not result in any outcome advantages as compared with lower levels of PEEP [41].

The authors hypothesized that the disappointing results of the recent clinical trial on PEEP setting led by the National Institutes of Health ARDS network [41] resulted from the enrolment and randomization of patients who had different degrees of lung recruitability. If so, higher PEEP could have been detrimental in patients who had lower lung recruitability, because the main effect would have been increased global stress and strain on the already open lung regions. On the other hard, in patients who had higher lung recruitability the lower PEEP could have been harmful, because it cannot extensively prevent the intratidal collapse and decollapse of alveoli, thereby allowing excessive regional stress and strain.

CT scanning has always seemed to be the best available technique for studying lung collapse and decollapse [42]. The authors recently studied the lung recruitability in 68 patients who had ALI/ ARDS by obtaining two whole-lung CT scans at $5 \mathrm{~cm} \mathrm{H}_{2} \mathrm{O}$ PEEP and at $45 \mathrm{~cm} \mathrm{H}_{2} \mathrm{O}$ airway plateau pressure, respectively [16]. Lung recruitability was defined as the lung tissue regaining aeration from 5 to $45 \mathrm{~cm} \mathrm{H}_{2} \mathrm{O}$ airway pressure and was expressed as a percentage of the total lung weight. Moreover, to assess the effects of the application of higher PEEP, they obtained a further CT scan of the whole lung at $15 \mathrm{~cm} \mathrm{H}_{2} \mathrm{O}$ PEEP. Physiologic variables in gas exchange (oxygenation, $\mathrm{PaCO}_{2}$, dead space, and other variables) and respiratory mechanics (respiratory system compliance) also were recorded after the PEEP trial at 5 and

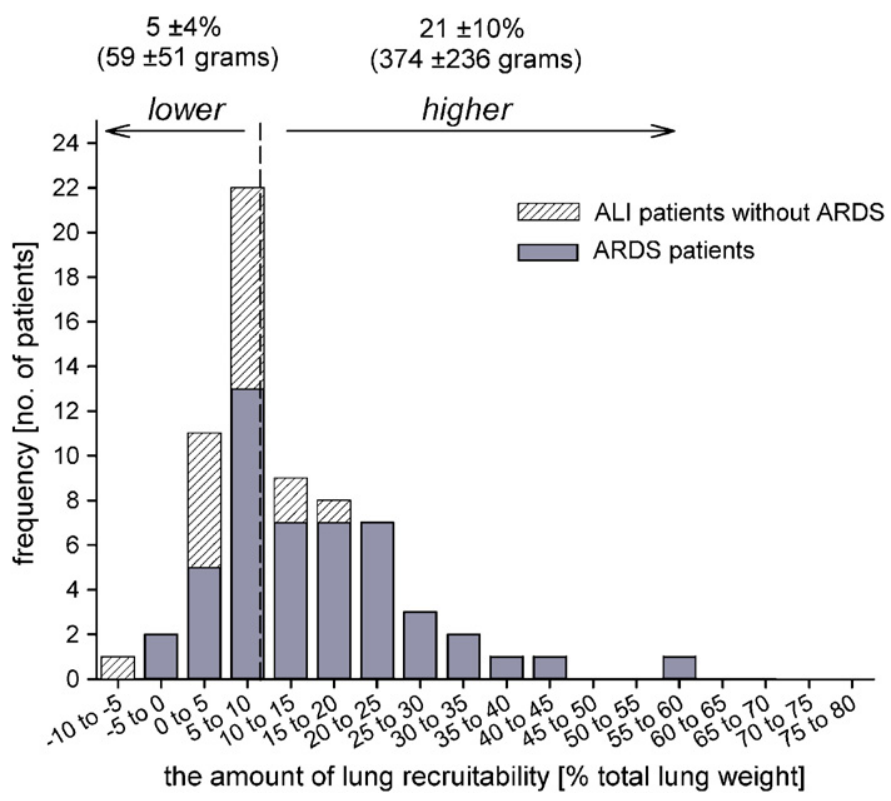

Fig. 4. The frequency distribution of the 68 patients in the overall study population according to the amount of the lung recruitability, expressed as the percentage of the total lung weight. ALI without ARDS was defined by a $\mathrm{PaO}_{2} / \mathrm{FIO}_{2}$ of less than 300 but not less than 200, and ARDS was defined by a $\mathrm{PaO}_{2} / \mathrm{FIO}_{2}$ of less than 200. The lung recruitability was defined as the proportion of lung tissue in which aeration was restored at airway pressures between 5 and $45 \mathrm{~cm}$ $\mathrm{H}_{2} \mathrm{O}$. ALI, acute lung injury; ARDS, acute respiratory distress syndrome; $\mathrm{PaO}_{2} / \mathrm{FIO}_{2}$, ratio of partial pressure of arterial oxygen to fraction of inspired oxygen. (From Gattinoni L, Caironi P, Cressoni M, et al. Lung recruitment in patients with the acute respiratory distress syndrome. N Engl J Med 2006;354(17):1780; with permission. Copyright (C) 2004 Massachusetts Medical Society, all rights reserved.) 
$15 \mathrm{~cm} \mathrm{H}_{2} \mathrm{O}$ PEEP to verify the possibility of estimating the lung recruitability at the bedside by using respiratory physiologic variables.

Fig. 4 presents the frequency distribution of lung recruitability in the study population. As shown, the lung recruitability in humans is far lower than that observed in experimental animal models (median value, $9 \%$ of the total lung weight). Moreover, the authors found that the application of $15 \mathrm{~cm} \mathrm{H}_{2} \mathrm{O}$ PEEP is able to maintain recruited about $50 \%$ of the recruitable lung, independent of its absolute amount. Unexpectedly, lung recruitability was strictly associated with the overall severity of lung injury, as detected by $\mathrm{CT}$ scan and by respiratory physiologic variables measured at the bedside, such as gas exchange and respiratory mechanics. Similarly, lung recruitability seemed to be closely associated with mortality. Of note, the respiratory physiologic variables assessed at the bedside, particularly the changes in oxygenation, were poor predictors of lung recruitability as assessed by CT scan.

On the whole, the use of CT scanning, with its quantitative analysis, revealed a new pathophysiologic aspect of ARDS, that is, the large variability in the amount of lung recruitability and its strong association with the response to PEEP. Moreover, it seems that CT scanning cannot be replaced by simple techniques available at the bedside, such as variations in oxygenation. At present, there are no alternatives to CT scanning to determine a given patient's lung recruitability, and it is quite evident that the assessment of the lung recruitability is necessary. For instance, the use of higher levels of PEEP in a patient who has lower lung recruitability does not have any physiologic rationale. In fact, a higher level of PEEP may maintain only recruited lung regions that are already open to aeration. It cannot keep open a significant fraction of the lung parenchyma, because the lung recruitability is actually negligible in such a patient (a few grams of the total lung weight). In contrast, the use of lower levels of PEEP in patients who have greater lung recruitability is potentially harmful, because it cannot prevent the intratidal opening and closing of a significant fraction of the lung (about $200 \mathrm{~g}$ in each breath). Indeed, while awaiting a randomized, clinical trial, which should be performed only in patients who have higher lung recruitability, the authors apply higher levels of $\operatorname{PEEP}\left(>15 \mathrm{~cm} \mathrm{H}_{2} \mathrm{O}\right)$ in patients who have greater lung recruitability and reserve lower levels of PEEP $\left(<10 \mathrm{~cm} \mathrm{H}_{2} \mathrm{O}\right)$ for patients who have lower lung recruitability.

\section{Summary}

The authors have investigated ARDS using CT scanning for more than 20 years. The unique ability of CT scanning to provide a quantitative approach to observing the lung parenchyma has made it possible to understand better the pathophysiology of ARDS. The authors believe that today CT scanning, in addition to its use in research, may be an essential tool allowing a rational setting of mechanical ventilation in patients who have ARDS. The assessment of lung recruitability should guide a correct setting of PEEP. Moreover, the quantification of the portion of the lung open to ventilation should lead to the application of a safe tidal volume, without excessive alveolar strain. Future studies will investigate the efficacy of these approaches on outcome.

\section{References}

[1] Ashbaugh DG, Bigelow DB, Petty TL, et al. Acute respiratory distress in adults. Lancet 1967;2(7511): $319-23$.

[2] Rommelsheim K, Lackner K, Westhofen P, et al. [Respiratory distress syndrome of the adult in the computer tomograph]. Anasth Intensivther Notfallmed 1983;18(2):59-64 [in German].

[3] Gattinoni L, Mascheroni D, Torresin A, et al. Morphological response to positive end expiratory pressure in acute respiratory failure. Computerized tomography study. Intensive Care Med 1986;12(3): $137-42$.

[4] Maunder RJ, Shuman WP, McHugh JW, et al. Preservation of normal lung regions in the adult respiratory distress syndrome. Analysis by computed tomography. JAMA 1986;255(18):2463-5.

[5] Gattinoni L, Pesenti A, Torresin A, et al. Adult respiratory distress syndrome profiles by computed tomography. J Thorac Imaging 1986;1(3):25-30.

[6] Gattinoni L, Pelosi P, Pesenti A, et al. CT scan in ARDS: clinical and physiopathological insights. Acta Anaesthesiol Scand Suppl 1991;95:87-94.

[7] Gattinoni L, Caironi P, Pelosi P, et al. What has computed tomography taught us about the acute respiratory distress syndrome? Am J Respir Crit Care Med 2001;164(9):1701-11.

[8] Gattinoni L, Biondetti P, Carlesso E, et al. CT ventilation imaging: technical background and impact in acute lung injury and ARDS management. In: Lipson DA, van Beek EJR, editors. Functional lung imaging. Lung biology in health and disease, vol. 200. Boca Raton (FL): 2005. p. 33-61.

[9] Mull RT. Mass estimates by computed tomography: physical density from CT numbers. AJR Am J Roentgenol 1984;143(5):1101-4. 
[10] Gattinoni L, Pesenti A, Bombino M, et al. Relationships between lung computed tomographic density, gas exchange, and PEEP in acute respiratory failure. Anesthesiology 1988;69(6):824-32.

[11] Ibanez J, Raurich JM. Normal values of functional residual capacity in the sitting and supine positions. Intensive Care Med 1982;8(4):173-7.

[12] Pelosi P, D'Andrea L, Vitale G, et al. Vertical gradient of regional lung inflation in adult respiratory distress syndrome. Am J Respir Crit Care Med 1994; 149(1):8-13.

[13] Gattinoni L, Pelosi P, Vitale G, et al. Body position changes redistribute lung computed-tomographic density in patients with acute respiratory failure. Anesthesiology 1991;74(1):15-23.

[14] Gattinoni L, Pesenti A, Avalli L, et al. Pressurevolume curve of total respiratory system in acute respiratory failure. Computed tomographic scan study. Am Rev Respir Dis 1987;136(3):730-6.

[15] Crotti S, Mascheroni D, Caironi P, et al. Recruitment and derecruitment during acute respiratory failure: a clinical study. Am J Respir Crit Care Med 2001;164(1):131-40.

[16] Gattinoni L, Caironi P, Cressoni M, et al. Lung recruitment in patients with the acute respiratory distress syndrome. N Engl J Med 2006;354(17): 1775-86.

[17] Murray JF, Matthay MA, Luce JM, et al. An expanded definition of the adult respiratory distress syndrome. Am Rev Respir Dis 1988;138(3): 720-3.

[18] Bernard GR, Artigas A, Brigham KL, et al. The American-European Consensus Conference on ARDS. Definitions, mechanisms, relevant outcomes, and clinical trial coordination. Am J Respir Crit Care Med 1994;149(3 Pt 1):818-24.

[19] Fink A, Kosecoff J, Chassin M, et al. Consensus methods: characteristics and guidelines for use. Am J Public Health 1984;74(9):979-83.

[20] Ferguson ND, Davis AM, Slutsky AS, et al. Development of a clinical definition for acute respiratory distress syndrome using the Delphi technique. J Crit Care 2005;20(2):147-54.

[21] Ferguson ND, Frutos-Vivar F, Esteban A, et al. Acute respiratory distress syndrome: underrecognition by clinicians and diagnostic accuracy of three clinical definitions. Crit Care Med 2005;33(10): 2228-34.

[22] Meade MO, Cook RJ, Guyatt GH, et al. Interobserver variation in interpreting chest radiographs for the diagnosis of acute respiratory distress syndrome. Am J Respir Crit Care Med 2000;161(1): 85-90.

[23] Gattinoni L, D'Andrea L, Pelosi P, et al. Regional effects and mechanism of positive end-expiratory pressure in early adult respiratory distress syndrome. JAMA 1993;269(16):2122-7.

[24] Schuster DP. What is acute lung injury? What is ARDS? Chest 1995;107(6):1721-6.
[25] Schuster DP. Identifying patients with ARDS: time for a different approach. Intensive Care Med 1997; 23(12):1197-203.

[26] Tagliabue M, Casella TC, Zincone GE, et al. CT and chest radiography in the evaluation of adult respiratory distress syndrome. Acta Radiol 1994; 35(3):230-4.

[27] Goodman LR. Congestive heart failure and adult respiratory distress syndrome. New insights using computed tomography. Radiol Clin North Am 1996;34(1):33-46.

[28] Desai SR, Hansell DM. Lung imaging in the adult respiratory distress syndrome: current practice and new insights. Intensive Care Med 1997;23(1):7-15.

[29] Weibel ER. Airways and blood vessels. The pathway for oxygen. Structure and function in the mammalian respiratory system. Cambridge (MA): Harvard University Press; 1984. p. 272-301.

[30] Ventilation with lower tidal volumes as compared with traditional tidal volumes for acute lung injury and the acute respiratory distress syndrome. The Acute Respiratory Distress Syndrome Network. N Engl J Med 2000;342(18):1301-8.

[31] Amato MB, Barbas CS, Medeiros DM, et al. Effect of a protective-ventilation strategy on mortality in the acute respiratory distress syndrome. N Engl J Med 1998;338(6):347-54.

[32] Brochard L, Roudot-Thoraval F, Roupie E, et al. Tidal volume reduction for prevention of ventilatorinduced lung injury in acute respiratory distress syndrome. The Multicenter Trail Group on Tidal Volume reduction in ARDS. Am J Respir Crit Care Med 1998;158(6):1831-8.

[33] Stewart TE, Meade MO, Cook DJ, et al. Evaluation of a ventilation strategy to prevent barotrauma in patients at high risk for acute respiratory distress syndrome. Pressure- and Volume-Limited Ventilation Strategy Group. N Engl J Med 1998;338(6):355-61.

[34] Brower RG, Shanholtz CB, Fessler HE, et al. Prospective, randomized, controlled clinical trial comparing traditional versus reduced tidal volume ventilation in acute respiratory distress syndrome patients. Crit Care Med 1999;27(8):1492-8.

[35] Gattinoni L, Carlesso E, Cadringher P, et al. Physical and biological triggers of ventilator-induced lung injury and its prevention. Eur Respir J Suppl 2003; 47:15S-25S.

[36] Pelosi P, Goldner M, McKibben A, et al. Recruitment and derecruitment during acute respiratory failure: an experimental study. Am J Respir Crit Care Med 2001;164(1):122-30.

[37] Muscedere JG, Mullen JB, Gan K, et al. Tidal ventilation at low airway pressures can augment lung injury. Am J Respir Crit Care Med 1994;149(5):1327-34.

[38] Webb HH, Tierney DF. Experimental pulmonary edema due to intermittent positive pressure ventilation with high inflation pressures. Protection by positive end-expiratory pressure. Am Rev Respir Dis 1974;110(5):556-65. 
[39] Dreyfuss D, Soler P, Basset G, et al. High inflation pressure pulmonary edema. Respective effects of high airway pressure, high tidal volume, and positive end-expiratory pressure. Am Rev Respir Dis 1988; 137(5):1159-64.

[40] Tremblay L, Valenza F, Ribeiro SP, et al. Injurious ventilatory strategies increase cytokines and c-fos m-RNA expression in an isolated rat lung model. J Clin Invest 1997;99(5):944-52.
[41] Brower RG, Lanken PN, MacIntyre N, et al. Higher versus lower positive end-expiratory pressures in patients with the acute respiratory distress syndrome. N Engl J Med 2004;351(4):327-36.

[42] Gattinoni L, Pelosi P, Crotti S, et al. Effects of positive end-expiratory pressure on regional distribution of tidal volume and recruitment in adult respiratory distress syndrome. Am J Respir Crit Care Med 1995;151(6):1807-14. 